\title{
VII.
}

\section{Die Würdigung des Fettpolsters der lateralen Tubenwand.}

\author{
Ein Beitrag zur Frage der Autophonie. \\ Von \\ Dr. Ostmann, \\ Privatdocent in Königsberg in Pr.
}

(Mit 8 Abbildungen.)

(Von der Redaction übernommen am 29. April 1892.)

In dem Bericht der Poliklinik für Ohrenkranke zu Tübingen theilt Wagen hä user ${ }^{1}$ ) drei Fälle mit, bei denen ,sich in schönster Reihenfolge Respirationsbewegungen am Trommelfell, auscultatorisch wabrnehmbares Athmungsgeräuseh und sehliesslich ausgesprochene Autophonie beobachten liessen". Diese Erscheinungen wurden von ihm auf abnormes Offenstehen der Tuba Eustachii zurückgeftuhrt. In allen drei Fällen bestand zur Zeit der Beobachtung Kräfteverfall, welcher in dem Falle, der uns hier besonders interessirt und einen 55 jährigen Mann betraf, im Gefolge einer starken, linksseitigen Infiltration der Wangenschleimhaut (Syphilis?) aufgetreten war. Hier hatten sich die ersten Symptome von Seiten des Ohres gleichzeitig mit der Affection der linken Wange entwickelt und waren mit der Verschlechterung des Allgemeinbefindens stetig gewachsen. Ein objectiv nachweisbarer krankhafter Befund wurde weder im Rachen, noch im Mittelohr gefunden. Die therapeutischen Maassnahmen hatten keinen oder einen nur ganz vorïbergehenden Erfolg - Einlegen dicker Bougies während längerer Zeit in die Tuba - ; die Autophonie verlor sich von selbst, nachdem sich der Mann innerhalb eines Monats ,trefflich erholt und gekräftigt" hatte, und das früher sehr lästige Brausen während der Respiration trat nur noch bei forcirtem Athmen auf. Den endguiltigen Ablauf der Erkrankung konnte Wagenhäuser zwar nicht beobachten, doch glaubt er

1) Archiv f. Ohrenheilk. Bd. XXI. S. 272. 
annehmen zu dürfen, „dass sich mit dem weiteren Heben des Allgemeinbefindens der Zustand vollständig zurückgebildet haben wird".

Ueber zwei weitere, uns hier interessirende Fälle hat Hart$\left.\operatorname{mann}{ }^{1}\right)$ auf der 56. Versammlung deutscher Naturforscher und Aerzte berichtet. Beide Male fand sich die Autophonie bei Individuen, bei denen durch eine kurz vorhergegangene acute Erkrankung (Pneumonie) bezw. im Verlaufe einer chronischen Lungenphthise sich ausgesprochene Schwächezustände entwickelt hatten, und Hartmann ist geneigt, diese und insbesondere die verminderte Muskelenergie - doch wohl der Tubenmuskeln? - als Ursache der abnormen Erscheinungen von Seiten des Ohres anzusehen. Angenommen, diese Erklärung für das Auftreten von Autophonie bei hochgradigen Schwächezuständen wäre richtig, so passt sie jedoch nicht für die Fälle, wo bei alten kräftigen Leuten, bei denen sich derartige Schwächezustände nicht nachweisen lassen, Autophonie auftritt (Wagen häuser).

Schliesslich berichtet Bezold ${ }^{2}$ ) uber doppelseitiges Offenstehen der Tuba bei einem Arzt, „der wegen einer schweren Magen- und Leberaffection in den letzten Monaten seine Kost anf Suppe und leicht verdauliche Gemüse beschränkt und infolgedessen sein Gewicht rasch um 40 Pfund und seinen Umfang um $.26 \mathrm{Cm}$. verringert hatte". Es wurde über ein eigenthimliches Zufallen der Ohren geklagt, während dessen jede stärkere Athembewegung ,wie in einem hohlen Rohr" wahrgenommen wurde, und die Sprache auffällig stark im Ohre erklang. Links bestanden Respirationsbewegungen des Trommelfells, ohne dass die Hörschärfe nennenswerth herabgesetzt war, was mit den Beobachtungen Lucae's ibereinstimmt, der bei offenstehender Tuba mehrfach normales Hörvermögen fand. „Rachen- und Nasenkatarrh fehlten vollständig."

Diesen Fällen kann ich einen eigenen anreihen, wo bei einem jungen Manne, der nach Ueberstehen eines acuten Gelenkrheumatismus und eines an diesen sich unmittelbar anschliessenden Unterleibstyphus hochgradig abgemagert war, während der Reconvalescenz etwa 14. Tage lang ausgesprochene Antophonie namentlich rechterseits bestand, die sich bei Hebung des Kräftezustandes und Erhöhung des Körpergewichts von selbst verlor.

Diese bisher allerdings sehr spärlichen klinischen Beobach-

1) Archiv f. Ohrenheilk. Bd. XX. S. 291.

2) Ebenda. Bd. XXI. S. 232. 
tungen haben das Gemeinsame, dass die eigenartigen Erscheinungen von Seiten des Ohres - Respirationsbewegungen am Trommelfell, Autophonie, starkes, vom Gehörgang aus wahrnehmbares Respirationsgeräusch - während starker Abmagerung anftraten und, sofern die Patienten am Leben blieben, bei Hebung der Kräfte, soweit darïber berichtet wird, von selbst schwanden, sowie dass eigentliche Erkrankungen des Gehörapparates selbst nicht nachgewiesen wurden. Man führte die Erscheinungen allgemein zurück auf ein abnormes Offenstehen der Tuba, welches, wie aus dem Kommen und Gehen der Erscheinungen hergeleitet werden muss, jedoch nur während der Zeit der Abmagerung und des Kräfteverfalls bestand. In der mit schweren Erkrankungen einhergehenden Abmagerung müssen wir also anscheinend die wesentliche Ursache für das zeitweise Offenstehen der Tuba suchen; es fragt sich nur, wie wir uns den Causalnexus zwischen beiden zu denken haben.

Die von Hartmann (1. c.) ausgesprochene Ansicht, es sei die mit dem Kräfteverfall einhergehende ,verminderte Muskelenergie" im Wesentlichen die Ursache der zeitweiligen Eröffnung, kömnen wir von vornherein als nicht stichbaltig bezeichnen, da, wenn schon bei normaler Energie die Tubenmuskeln ein Offenstehen der Tuba nicht herbeiführen, sie nach der Art und Weise ihrer Wirkung bei verminderter Energie noch viel weniger eine dauernde Eröffnung derselben herbeiführen werden. Die Schwächung der Tubenmuskeln kann einzig und allein ein erschwertes Oeffnen der Tuba zur Folge haben. Der innere $\mathrm{Zu}$ sammenhang muss also ein anderer sein.

Unter den Geweben der lateralen häutigen Tubenwand findet sich eins, das Fettgewebe, welches bei verminderter Nahrungsaufnahme und erhöhtem Kräfteverbranch, wie die alltägliche Erfahrung lehrt und die Untersuchungen Flemming's ${ }^{1}$ ) wissenschaftlich nachweisen, sehr schnell und von allen Geweben im umfangreichsten Maasse schwindet. In diesem das vermittelnde Glied zwischen Abmagerung und Offenstehen der Tuba zu suchen, dürfte um so näher liegen, als anch bei Greisen, bei denen das Fett, ohne dass krankhafte Störungen vorzuliegen brauchen, physiologisch mehr oder weniger zu schwinden pflegt, von $R \ddot{u}-$ dinger2) ein Offenstehen der Ohrtrompete in ibrem ganzen

1) Ueber Bildung und Rückbildung der Fettzelle im Bindegewebe u. s. w. Archiv f. mikroskopische Anatomie. Bd. VII. S. $32 \mathrm{ff}$.

2) Monatsschr. f. Ohrenheilk. 1868. Nr.9. 
Verlanf wie sonst nur bei der Eröffnung gefunden wurde, and Wagenhäuser (l. c.) bei einem alten, sonst völlig gesunden und kräftigen Manne Autophonie infolge abnormen Offenstehens der Tuba beobachten konnte.

Um den angenommenen Causalnexus zu erweisen, fragt es sich zunächst, ob überhaupt allein durch Offenstehen der Tuba bei völlig gesundem Gehörapparate Autophonie erzeugt werden kann.

Die Bezeichnung Autophonie ist nicht gïnstig gewählt; denn unsere eigene Stimme hören wir stets; wir verstehen aber unter dieser Bezeichnung nur eine veränderte Perception derselben, welche darin besteht, dass unsere Stimme, die für gewöhnlich anscheinend vor dem Munde entsteht, innerhalb des Kopfes zu ertönen scheint, indem sie gleichzeitig höher und mit anderer Klangfarbe unter unangenehmen Dröhnen wahrgenommen wird. Nicht immer dürfte es leicht sein, zu sagen, ob bei einem Kranken, der über ein Dröbnen bei der Stimmgebung klagt, gerade dieser Symptomencomplex vorliegt, da die bezüglichen Angaben nur allzu sehr dem subjectiven Érmessen unterworfen sind.

In den uber Autophonie erschienenen Arbeiten 1) werden mehrfache Ursachen für dieselbe angegeben, und die Erklärungen für den einzelnen Fall stehen sich auch wohl einmal direct gegenuiber. Was der Eine durch Eröffnung der Tuba erklären will, erklärt der Andere durch ihren Verschluss. Eins dürfte indess unzweifelhaft feststehen, dass bei weit geöffneter Tuba Autophonie entstehen kann.

Derartige einwandfreie Beispiele finden sich mehrfach verzeichnet. So berichtet Hinton ${ }^{2}$ ) von Juile, dass er die Fähig-

1) Rüdinger, Ueber das Hören der eigenen Stimme durch die Tuba Eastachii. Monatsschr. f. Ohrenheilk. 1872. Nr. 9. - Transactions of the american otological Society seventh annual meeting. Newport, R. J. Juli 15. 1874. Referat im Archiv f. Ohrenheilk. Bd.X. S. 70 u. f. - Flemming, Notiz zur Beurtheilung des normalen Situs der Fustachischen Röhre. Monatsschr. f. Ohrenheilk. 1875. Nr. 6. Referat im Archiv f. Ohrenheilk. Bd. X. S. 259 u.f. - Gruber, Ueber Autophonie and Tympanophonie. Nonatsschr. f. Ohrenheilk. Jahrg. I. Nr. 8. - Brunuer, Zur Aetiologie und Symptomatologie der sogenannten Autophonie. Zeitschr. f. Ohrenheilk. Bd. XII. 4. S. 268. Referat im Archiv f. Ohrenheilk. Bd. XX. S. 208. - E. Berthold, Ueber die Autophonie. Revue mens. d'Otologie. April 1884. Referat im Archiv f. Ohrenheilk. Bd. XXII. S. 97 und Separatabdruck - und andere kurze Mittheilungen.

2) Referat im Archiv f. Ohrenheilk. Bd. X. S. 211. 
keit besessen habe, willkürlich seine Ohrtrompete zu öffnen, wobei die Töne mit verstärkter Intensität gehört wurden; Flemming (l. c.) vermochte nach Belieben durch eine "schwer zu definirende Muskelbewegung" die Tuba zu eröffnen und dadurch starke Autophonie hervorzubringen, desgleichen Zaufal (l. c.) während des ersten Actes einer Gähnbewegung; Kir chner I) kann willkürlich Autophonie herbeifuhren, wenn er das Gähnen unterdrückt.

In diesen Fällen blieben alle ïbrigen Verhältnisse unverändert, nur die Tuben warden willkürlich eröffnet, und es entstand Autophonie. Wir können demnach auch keinen Anstand nehmen, dieselbe durch die Eröffunng allein bedingt anzusehen.

Bei hochgradigen Abmagerangszuständen im mittleren Lebensalter eröffnet sich nun die Ohrtrompete durch Schwund des Fettpolsters, vornehmlich an ihrer lateralen, häutigen Wand.

Zum Nachweis hierfür fragen wir:

1. Wie verhält sich das Fettpolster unter normalen Ernährungsverhältnissen?

2. Wie verbält es sich bei starker Abmagerung?

3. Wie erklärt sich die Eröffnung der Tuba beim Schwund desselben?

Das Gewebe der lateralen Tubenwand ist nach $\mathrm{Henle}{ }^{2}$ ), in der oberen Hälfte ziemlich fest, aus verflochtenen Bindegewebsbündeln zusammengesetzt, in der unteren Hälfte dagegen eine lockere, schwammige Substanz, deren Hauptbestandtheil Fett ausmacht", und auf der beigegebenen Abbildung (582) erstreckt sich das Fettpolster vom Tubenboden nach unten und aussen, so dass die laterale Tubenwand selbst ganz frei von eingelagertem Fett erscheint. Hyrtl erwähnt in seinem Lebrbuche der Anatomie das Fettpolster gar nicht. Rü din $\mathrm{ger}^{3}$ ) bemerkt, dass das stark entwickelte Bindegewebsnetz, welches sich an der lateralen Tubenwand in der Nähe der knöchernen Tuba unter den Knorpelhaken findet und als ,ergänzende starre Umrahmung" der Ohrtrompete dient, weiter nach unten (,noch tiefer") durch ein Fettlager ersetzt werden kann. Betrachtet man die Abbildungen mit besonderer Rücksicht auf die Darstellung des Fettpolsters (Nr. 6, 7, 8, 9, $11,14,15,16)$, so zeigt sich einmal, dass sich dasselbe nicht

1) Archiv f. Ohrenheilk. Bd. XX. S. 291.

2) Handbuch der Eingeweidelehre des Menschen. S. 755. Braunschweig 1866.

3) Beiträge zur vergleichenden Anatonie und Histologie der Ohrtrompete. S. 8. München 1870. 
allein im unteren pharyngealen Abschnitt, sondern anch im mittleren und oberen Drittel der häutigen Wand (Fig. 9, 11, 14, 15, 16) und zwar in sehr kräftiger Entwicklung finden kann, denn auf dem Querdurehsebnitt 9 durch das obere Drittel der Tuba sieht man, wie es in der Erklärung zu der Abbildnng heisst, nur Fett; sodann aber zeigt sich auch, dass die Fetteinlagerung mannigfachen individuellen Schwankungen unterliegt. Wo es sich findet, liegt es in dem losen Bindegewebsgerüste der lateralen Wand und schiebt sich keilförmig zwischen den Musculus tensor und Levator veli palatini gegen die Schleimhaut der Tubenspalte vor. In ganz ähnlicher Weise wie beim Menschen findet sich auch beim Reh, Schaf, Kalbe, Ochsen, Schwein und bei der Ziege ein in seiner Mächtigkeit bei den einzelnen Thieren wechselndes Fettlager und zwar nicht allein an der lateralen, sondern zum Theil auch an der medialen Wand. Nach Schwalbe ${ }^{1}$ ) ist die Submucosa des rein membranösen Abschnittes der lateralen Tubenwand „häufig in ihrer ganzen Höhe durch eine bis $11 / 2 \mathrm{Mm}$. dicke Fettschicht vertreten, welche nach dem Bodenende der lateralen Wand ihre grösste Dicke besitzt, nach dem Knorpelhaken zu allmählich an Dicke abnimmt". Merkel'2), Politzer ${ }^{3}$ ) und v. Tröltsch äussern sich in ähnlicher Weise wie die vorgenannten Autoren über das Vorkommen von Fett an der bezüglichen Stelle.

Wenn man sich darüber klar werden will, in welcher Ausdehnung sich das Fettpolster bei normal entwickelten and ernährten Personen mittleren Alters im Allgemeinen findet, so wird man mit Rücksicht auf die schnelle Rückbildung des Fettes bei verminderter Nahrungsaufnahme und Einwirkung anderer, die Ernährung des Körpers beeinträchtigender Ursachen nicht die Tuben solcher Personen untersuchen dürfen, die erst nach längerer Erkrankung, sondern die eines plötzliches Todes gestorben sind. Umgekehrt werden die Veränderungen, welche das Fettpolster bezïglich seiner Ausdehnung und seines sonstigen Verhaltens bei Abmagerung erleidet, am schärfsten an Tuben hervortreten, welche hochgradig abgemagerten Leichen entnommen sind.

Das diesen Anforderungen entsprechende Material, welches mir in der letzten Zeit zu Gebote stand, ist klein; es beschränkt sich auf je 2 Fälle.

1) Anatomie der Sinnersorgane. S. 534.

2) Handbuch der topographischen Anatomie. Bd. I. S. 577 u. 579.

3) Lehrbuch der Ohrenheilkunde. S. 34. 2. Aufl. 
Die Untersuchungsmethode war folgende.

Die mit ihrer Umgebung herausgenommenen Tuben wurden zunächst unzerschnitten 24 Stunden in Flemming'sche Lösung gelegt. Nachdem sie hierdurch eine gewisse Festigkeit erhalten hatten, so dass das Anlegen glatter, zur Längsaxe der Tuba senkrecht gestellter Schnitte leichter möglich war, wurden dieselben in eine Serie 2-3 Mm. dicker Schnitte zerlegt and diese wiederum 1-2 Tage in die gleiche Lösung gelegt. Es hatte dann einerseits eine genügende Erhärtung, andererseits eine vollkommene Schwarzfärbung des auf den Schnittflächen zu Tage tretenden Fettes stattgefunden, so dass sich die Ausdehnung und Lagerung desselben schon mit unbewaffuetem Auge, noch schärfer bei Lupenvergrösserung mit voller Bestimmtheit erkennen liess. Aus den einzelnen Tubenabschnitten wurden dann behufs mikroskopischer Untersuchung des Fettes mit dem Mikrotom feinere Schnitte angefertigt. Die ersten der von mir untersuchten Tuben, welche einer der hochgradig abgemagerten Leichen entstammten, sind indess einer anderen Behandlungsmethode unterworfen, da ich die Vorzitge der erstangegebenen, welche sich zur schnellen Darstellung des in das Gewebe eingestrenten Fettes vorzüglich eignet, noch nicht kannte. Diese Tuben wurden 3 Tage in 96 proc. Alkohol und 24 Stunden in absolutem Alkohol erhärtet, dann in der gleichen Weise wie zuvor zerschnitten und in 1 proc. Osmiumsäure gefärbt. Um dem Einwande zu begegnen, dass das Fett durch das Einlegen der Tuben in absoluten Alkohol ausgezogen worden sei, habe ich ein bohnengrosses Stück Fett aus dem Unterhautzellgewebe einer mässig fettreichen Leiche 7 Tage in 96 proc. und 36 Stunden in absolutem Alkohol erhärtet und dann die Mikrotomschnitte in Osmiumsäure gefärbt and in Glycerin untersucht. Ich habe in keinem Schnitt halb- oder ganz leere Fettzellen gefunden, vielmehr waren dieselben durchgehends bis zur vollen Rundung mit dem tiefschwarzgefärbten Fett gefüllt, so dass ich auch jede störende Einwirkung der Methode auf das Untersuchungsergebniss des Fettpolsters der Tube ausschliessen darf.

Dasselbe ist folgendes.

Betrachten wir zunächst die Durchschnitte der Tuben, welche der Leiche eines 42 jährigen, kräftìg gebauten, im Allgemeinen fettarmen Mannes entnommen sind, der eines plötzlichen Todes gestorben war. Auf denjenigen durch das obere, der knöchernen Ohrtrompete zunächst gelegene Tubendrittel fand sich in der 
ganzen Höhe der lateralen Wand zwischen der Schleimbaut der Tubenspalte und dem Museulus tensor veli palatini ein 1-11/2 Mm. dickes Fettpolster, welches sich nach unten und lateralwärts als ein feiner Streifen zwisehen den Tensor und Levator veli palatini hineinschob (siehe Fig. 1). Ausserdem war in das Gewebe der medialen Wand, sowohl zwischen Tubenknorpel nnd Schleimhaut, wie unterhalb des ersteren, Fett in Form kleinerer und

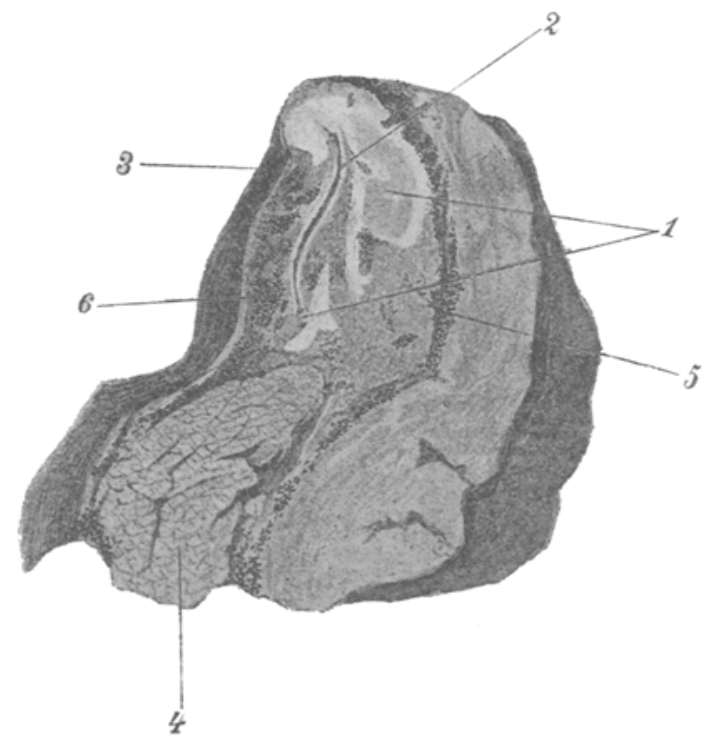

Fig. 1. Durchsehnitt duroh das obere Tabendrittel. 1 Tubenknorpel (accessorischer Knorpel am Tubenboden). 2 Tubenspalte. 3 Musculus tensor veli palatini. 4 Musculus levator veli palatini. 5 Fettpolster an der medialen Tubenwand. 6 Fettpolster an der lateralen Tubenwand.

grösserer Nester eingestreut. Schliesslich fand sich an der me. dialen Fläche des Tubenknorpels ein zusammenhängender Fettstreifen, welcher sich nach unten bis an die mediale Fläche des Musculus levator veli palatini verfolgen liess, an dieser Stelle jedoch weniger zusammenbängend wurde. Im mittleren und pharyngealen Drittel der knorpligen Tuba verschwand das Fettpolster der lateralen Tubenwand mehr und mehr, so dass man an Stelle desselben auf Schnitten, die dicht hinter dem Ostium tubae pharyngeum hindurchgelegt waren, nur noch vereinzelte kleine, in das Gewebe eingestreute Fettklümpchen wahrnahm (siehe Fig. 2), während sowohl das Fettpolster zwischen dem 
Levator and Tensor veli palatini, wie auch in der Umgebung des medialen Tubenknorpels an Mächtigkeit zugenommen batte. Auf den Durchschnitten dureh die Tuba eines sehr kräftig gebauten, musculösen, 22 jährigen jungen Mannes mit kaum mittelstark entwickeltem Fettpolster, der infolge eines Unglüeksfalles plötzlich verschieden war, war die Anordnung und Ausdehnung des Fettgewebes in der Umgebung der Ohrtrompete insofern eine

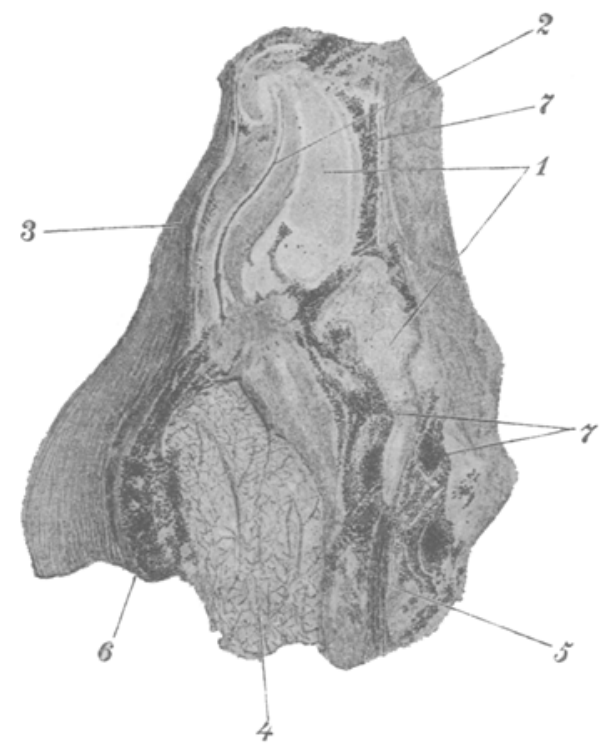

Fig. 2. Durehschnitt durch das untere Drittel derselben Tuba.

1 Tubenknorpel. 2 Tubenspalte. 3 Musculus tensor veli palatini.

4 Museulus levator veli palatini. 5 Musculus salpingo-pharyngeus.

6 Laterales Fettpolster zwischen Muss. tensor und levator, 7 Fettpolster an der medialen Tubenwand.

verschiedene, als im oberen Tubendrittel das laterale Fettpolster nicht ganz so mächtig wie im ersteren Fall, dagegen im mittleren und unteren Drittel sowohl zwischen dem Tensor und Levator, als auch an der medialen Wand noch etwas stärker entwickelt war. Bei der mikroskopischen Untersuchnng der den einzelnen Tabenabschnitten entnommenen Schnitte zeigten sich die Zellen des Fettgewebes stets mit Fett gefüllt; Andeutungen von Fettschwund waren nicht vorhanden.

Ganz anders stellen sich die Verhältnisse bezigglich des makroskopischen wie mikroskopischen Verhaltens des Fettpolsters der Tuba bei hochgradiger Abmagerung. 
Die Abbildungen 3 und 4 sind Durchschnitte durch das obere, bezw. untere Drittel der knorpligen Tuba eines 22 jährigen Mannes, dessen in gesunden Tagen ca. 60 Kilo betragendes Körpergewicht 8 Tage vor seinem Tode auf 39,5 Kilo zurïckgegangen war.

Bei Betrachtung der Abbildungen fällt sofort die ausserordentlich geringe Menge des in der nächsten Umgebung der Tubenspalte befindlichen Fettes auf. Lateralwärts fand sich nur noch

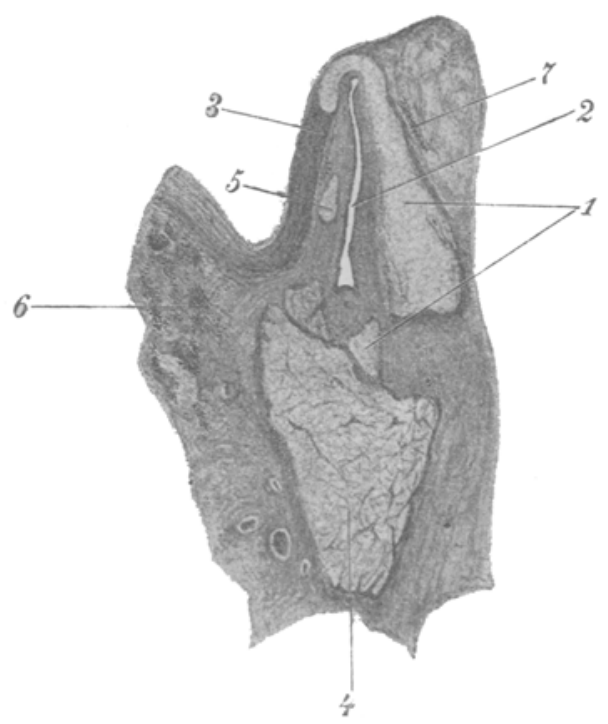

Fig. 3. Durchschnitt durch das obere Drittel der Tuba eines jungen Mannes bei starker Abmagerung.

1 Tubenknorpel (aceessorischer Tubenknorpel). 2 Tubenspalte (klaffend). 3 Museulus tensor veli palatini. 4 Muscalus levator veli palatini. 5 Knorpel in der lateralen, häutigen Tubenwand. 6 Fettpolster (stark geschwunden) zwisehen dem Musc. tens. und levator.

7 Fettgewebe an der medialen Seite des Tubenknorpels.

zwischen den Gaumen-Tubenmuskeln eine deutlichere Anhäufung von Fett, während an der Innenseite des medialen Tubenknorpels nur im oberen Tubenabschnitt ein feiner, schwarzgefärbter Fettstreifen (Fig. 3, 7) sichtbar war. Durch Schwund des Fettes an der lateralen Wand war das Gewebe so gelockert, dass es auf 2-3 Mm. dicken Durchschnitten tief einsank, und so zwischen der Tubenspalte und dem Musculus tensor und levator eine deutliche Grube entstand.

Die mikroskopische Untersuchung des Fettes sowohl von der lateralen wie medialen Tubenwand zeigte einen durch alle Stadien nachweisbaren Fettsehwund. 
Man sah einerseits Zellen, welche noch vollkommen oder doch bis auf einen schmalen, mehr grau gefärbten, peripheren

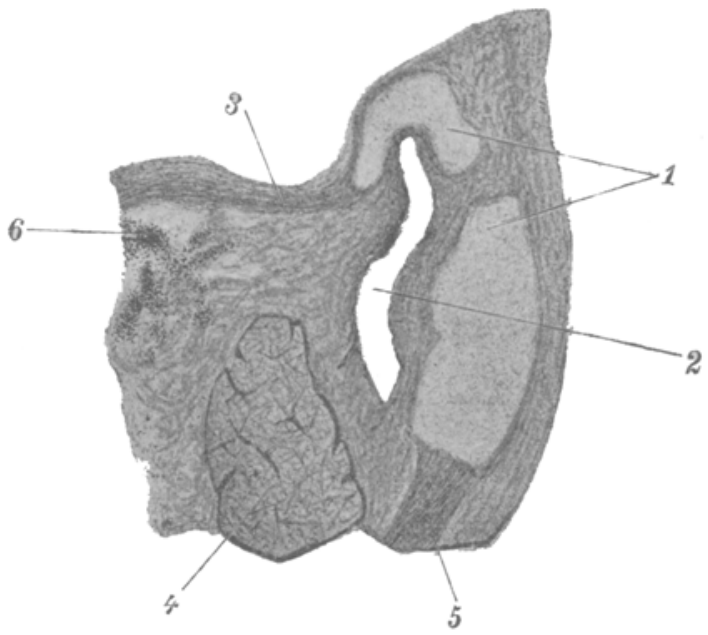

Fig. 4. Durehschnitt dureh dieselbe Tuba im unteren Drittel.

1 Tubenknorpel (getheilt). 2 Tabenspalte (klaffend). 3 Museulus tensor veli palatini. 4 Musculus levator veli palatini. 5 Musculus salpingo-pharyngeus. 6 Stark gesehwundenes Fettpolstex an dex lateralen Tubenwand.

Ring mit Fett gefüllt waren (siehe Fig. 5), daneben zum grössten Theil solche, in denen zumeist nicht ganz central eine oder zwei verschieden grosse Fettkugeln lagen, welche die etwas geschrumpfte

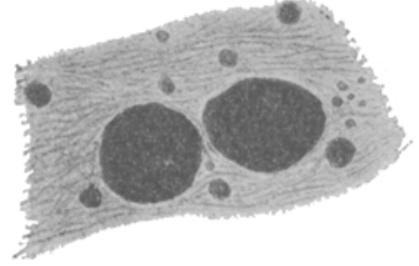

Fig. 5.

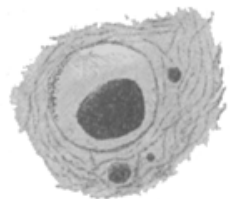

Fig. 6.

Zelle kaum zur Hälfte oder zu einem Drittel ausfüllten, während der übrige Zellraum entweder ganz leer war, oder nur eine verschieden grosse Anzahl kleinster wandständiger, durch Einwirkung der Osmiumsänre mehr oder weniger intensiv grangefärbter Fettkügelchen enthielt (siehe Fig. 6). In anderen Zellen hatte sich das Fett noch weiter zuriuckgebildet, und man fand an der mehrfach gefalteten Zellmembran nur Häufchen kleinster Fetttröpfchen angelagert, sowie häufig neben diesen im Innern der Zelle Ge- 
bilde, welche wie unregelmässig gestaltete, blassgraue Scheiben aussahen und aus Fetttröpfchen bestanden (siehe Fig. 7). Schliess-

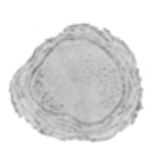

Fig. 7.

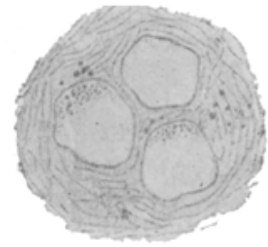

Fig. 8.

lich konnte man zahlreiche Zellen sehen, welche vollkommen leer waren und stark geschrumpft erschienen (siehe Fig. 8). In dem die Fettzellen einschliessenden Gewebe fanden sich vielfach Fettkïgelchen eingestrent, deren Grösse wie Intensität der Färbung vom tiefen Schwarz bis zum helleren Grau wechselte.

In beiden untersuchten Fällen von hochgradiger Abmagerung war der mikroskopische Befund der nämliche. Es kann demnach keinem Zweifel unterliegen, dass in der Umgebung dieser Tuben sich in gesunden Tagen eine grössere Menge von Fett angelagert hatte, welches bei der allgemeinen Abmagerung, die im Verlaufe der tödtlichen Erkrankung eintrat, gleichfalls schwand. Dieser Vorgang wird sich in allen Fällen allgemeiner Abmagerung in mehr oder weniger ausgedehntem Umfange abspielen.

Es fragt sich daher, wie wir uns unter Beriucksichtigung der Wirkung der Tubenmuskeln eine durch Schwund des Fettpolsters bedingte zeitweilige Eröffnung der Tubenspalte zu erklären haben, und weshalb, wenn die Bedingungen zur Eröffnung der Tuba durch Fettschwund gegeben sind, nicht sehr viel häufiger bei Abmagerung Autophonie auftritt.

Die zahlreichen Untersuchungen, welche von Eustachius, dem ersten Beschreiber der Tuba, an bis jetzt von v. Tröltsch 1), Rüdinger ${ }^{2}$ ), Zuckerkandl ${ }^{3}$ ), Mayer ${ }^{4}$ ), v. Kostanecki ${ }^{5}$ ),

1) Beiträge zur anatomischen und physiologischen Würdigung der Tubenund Ganmenmusculatur. Archiv f. Ohrenheilk. Bd. I. S. 15 u. f., sowie Beiträge zur vergleichenden Anatomie der Ohrtrompete. Ebenda. Bd. Il. S. 214 u. f.

2) Beiträge zur vergleichenden Anatomie und Histologie der Ohrtrompete. München 1870 .

3) Zur Anatomie und Physiologie des Tuba Eustachiana. Monatsschr. f. Ohrenheilk. 1873. Nr. 12.

4) Studien über die Anatomie der Canalis Kustachii. München 1866. Referat im Archiv f. Ohrenheilk. Bd. III. S. 244.

5) Zur Morphologie der Tubengaumenmusculatur. Archiv f. Anatomie u. Physiologie. Anatom. Abth. 1891. II. u. III. Heft. 
Urbantschitseh 1) u. A. über das anatomische Verhalten und die physiologische Wirkung der Tubengaumenmusculatur angestellt worden sind, haben diese viel erörterte Frage zum Abschluss gebracht.

Wir kennen als Tubengaumenmuskeln den Tensor veli palatini seu Dilatator tubae, welcher von dem lateralen Haken des Tubenknorpels und mitunter mit einigen Fasern auch von dem membranösen Theil der lateralen Tubenwand entspringt; sodann den Levator veli palatini, der zumeist dicht unter dem Boden der Tubenspalte entlang zieht und dessen sehnige Ursprünge im Bereich des hinteren oberen Tubenabschnittes „einerseits aus dem Bindegewebe der Schleimhantbekleidung der medialen Knorpelplatte sich entwickeln, andererseits durch Bindegewebszïge mit dem Fettpolster der lateralen Wand in Verbindung stehen". ${ }^{2}$ ) Als dritter gesellt sich der Musculus salpingo-pharyngeus hinzu. Dieser entspringt im Bereich des pharyngealen Tubenabschnittes einerseits von dem unteren Ende der medialen Knorpelplatte, andererseits tritt er mit der in der Nähe befindlichen Schleimhaut in directe Verbindung und zieht nach hinten und unten zur hinteren Rachenwand. Physiologisch wichtig sind noch zwei Ligamente, da sie durch ihre Verbindung mit den Tubenmuskeln die Eröffnung der Ohrtrompete zu fördern geeignet sind: das Ligamentum salpingo-palatinum anterius, welches, wie Urbantschitsch nachwies, mit der Sehne des Musculus tensor veli palatini in Verbindung tritt und dadurch diesem Muskel, wie Zaufal zuerst vermuthete, einen directen Einfluss auf die Bewegungen der vorderen Tubenlippe ermöglicht, sowie das Ligamentum salpingo-pharyngeum, welches als Ersatz für Muskelfasern auftritt und durch seine Verbindung mit dem Museulus palatinopharyngeus die Wirkung des salpingo-pharyngeus zu unterstiitzen befähigt ist. Wenn diese Muskeln und Bänder gemeinsam wirken, so wird die Tuba eröffnet, indem die laterale von der medialen Wand abgezogen wird. Einen Muskel, der durch seine Contraction eine Verengerung des Tubenlumens herbeizuführen im Stande wäre, kennt man beim Menschen nicht. Es ist nun aber wohl die Frage berechtigt, durch welche anatomischen Verhältnisse der für gewöhnlich leichte Verschluss der Tuba denn überhaupt gewährleistet ist. Einmal dureh die Schleimhautfalten am

1) Zur Anatomie der Tuba Eustachii des Menschen. Referat im Archiv f. Ohrenheilk. Bd. X. S. 262.

2) Schwalbe, Anatomie der Sinnesorgane. S. 541. 
Boden der Hülfsspalte und zwischen dieser und der Sicherheitsröhre, sodann durch das Fettpolster der lateralen Tubenwand, vermittelst dessen der Tensor veli palatini, der durch die Belastung mit dem Gaumensegel sich auch in der Ruhe in einem gewissen Zustande der Spannung befindet, die Schleimhant der lateralen Wand gegen die der medialen andrückt. Wie wenig fest dieser Verschluss im Allgemeinen ist, erkennt man darans, dass schon ein Vorwärtsneigen des Kopfes sowohl das Experimentum Vasalsae, wie den Katheterismus der Tuba leichter gelingen lässt, indem durch diese Lageveränderung des Kopfes die laterale Tubenwand dureh ihre eigene Schwere, wie durch die des mit ihr durch den Musculus tensor veli palatini verbundenen Gaumensegels bis zu einem gewissen Grade abgehoben wird.

Schwindet nun das Fettpolster der lateralen Tubenwand, wie wir es für hochgradige Abmagerungszustände nachgewiesen haben, so wird dieselbe noch nachgiebiger und schlaffer, als sie es schon an und fur sich ist, sie sinkt, ihrer elastischen Unterlage beraubt, zurück und es wird verständlich, wie unter diesen Umständen der Musculus tensor tympani infolge des Zusammen-

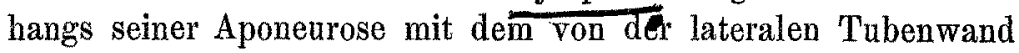
zur Mittellinie des harten Gaumens hinziebenden fibrösen Gewebe und der mehrfach beobachteten directen Verbindung seiner Fasern mit dem submucösen Gewebe gerade im oberen Tubendrittel, wo das Lamen am engsten ist, im Stande ist, auch in der Ruhe durch seine natürliche Spannung die laterale Tubenwand von der medialen abzuziehen und damit die Tuba dauernd zu eröffnen. In ähnlicher Weise ziehen die Augenmuskeln den Augapfel in die Orbita zuriick, sobald das Fettpolster, auf dem derselbe ruht, schwindet, während er, sobald das geschwundene Fett sich wieder bildet, anch in seine ursprüngliche Lage zurückkehrt.

Ebenso wird der in gesunden Tagen bestandene Verschluss der Tube wiederhergestellt, sobald bei der wieder eintretenden Fettbildung das Fettpolster der Tuba sich regenerirt.

Damit erklärt sich das Auftreten der Autophonie und der übrigen auf eine Eröffnung der Tuba zurückzufübrenden abnormen Erscheinungen während starker Abmagerung, wie das Verschwinden derselben bei Besserung des Kräfte- und Ernährungszustandes.

Diese Erklärung wird durch den naheliegenden Einwand nicht entkräftet, dass dann in der Reconvalescenz nach schweren Er- 
krankungen, die ja stets eine Abmagerung herbeiführen, sehr viel häufiger störende Autophonie beobachtet werden muisste.

Dem gegenüber ist zu bedenken, dass das Fettpolster eine individuell verschiedene Entwicklung zeigt und selbst bei der hochgradigsten Abmagerung, wie in unseren Fällen, nicht vollkommen schwindet, sowie dass die directe Verbindung zwischen dem Musculus tensor veli palatini und der häutigen Tubenwand durchaus nicht immer sich findet, sondern individuell verschieden gestaltet, somit auch der Muskel nicht in allen Fällen den gleichen unmittelbaren Einfluss auf die laterale, häutige Tubenwand gewinnen wird. Sodann wird die Erweiterung, welche das Tubenlumen durch Schwund des Fettpolsters erfäbrt, compensirt werden können und in sehr vielen Fällen auch ausgeglichen werden durch Schwellungen der Rachen- und Tubenschleimhaut, wie solche sich im Gefolge sehwerer Allgemeinerkrankungen, sofern sie nicht schon zuvor bestanden, sehr häufig entwickeln; auch kann durch Ansammlung von Schleim im Ostium tubae pharyngeum und im Tubenkanal selbst, wie dies ja ansserordentlich häufig beobachtet wird, das Lamen verlegt sein, oder infolge der mit dem Kräfteverfall einhergehenden Schwächung der Tubengaumenmuskeln ein Abziehen der Tubenwunde trotz des theilweisen Schwundes des Fettpolsters hintangehalten werden. Schliesslich dürfte die Zahl der bisher veröffentlichten Fälle auch deshalb so gering sein, weil man diesen vorübergehenden und von keiner merkbaren Schwächung des Hörvermögens begleiteten Erscheinungen von Seiten des Ohres während der Reconvalescenz nach schweren Erkrankungen da, wo sie sich einmal finden, wenig Beachtung gesehenkt haben durfte. Sie werden am leichtesten da auftreten, wo während hochgradiger Abmagerung Rachen- und Tubenschleimhaut vollkommen gesund sind, und in zwei der veröffentlichten Fälle wird dies auch besonders aufgeführt. Erwähnt sei noch, dass auch die Schwächung der Stimme während des Kräfteverfalls dem Auftreten störender Autophonie nicht günstig ist, und dass dasselbe auch davon bis zu einem gewissen Grade beeinflusst werden dürfte, ob die Stimme eine volltönende und klangreiche ist oder nicht.

Die Antophonie ist indess nur eins der durch die Eröffung der Tuba hervorgerufenen Symptome. Unter der Voraussetzung, dass das Tubenlumen frei ist, müssten das vom äusseren Gehörgang aus wahrnehmbare Respirationsgeräusch, sowie die bei der Respiration im Ohrmanometer sichtbaren Schwankungen gegen- 
iiber dem normalen Verhalten verstärkt werden, ebenso eventuell Respirationsbewegungen zumal am krankhaft veränderten Trommelfell (Atrophien, Narben) besonders deutlich hervortreten.

Ich habe versucht, mir ein eigenes Urtheil zu bilden, wie sich zunächst das normale Ohr bezïglich dieser Punkte verhält, und habe zu diesem Zweck eine grössere Reihe normal hörender junger Leute (zugewandte Flüstersprache, leicht verständliche Worte, wie Soldat, oder Zahlen 2, 6, 30, 72 u. s. w. in 20 bis 25 Meter) darauf hin untersucht. Bei diesen Versuchen zeigte sich, dass man bei jungen Leuten mit guter Athmungsbreite (7-10 Cm.), zuweilen schon bei ganz rubiger Athmung durch die Nase, gar nicht selten ein in- wie exspiratorisches Athmungsgeräusch vom äusseren Gehörgange als ein feines, weiches Hauchen wahrnimmt, welches bei den einzelnen Personen in seiner Intensität wechselt und bei der Ausathmung häufig einen hohlen Beiklang hat. Das Ergebniss der Manometeruntersuchungen, welche genau nach der von $L u c a$ angegebenen Weise angestellt wurden, war auch ein schwankendes; denn ich konnte fast constant etwa 1/2 Mm. betragende, mit dem Puls isochrone, indess durchaus nicht immer mit der Respiration zusammenfallende Manometerschwankungen wahrnehmen. Deutliche Respirationsbewegungen habe ich am normalen Trommelfell niemals, wohl aber, wie Schwartze und Lucae, an Trommelfellnarben gesehen, wobei sich dieselben während der Einathmung gegen die Trommelhöhle und während der Ausathmung gegen den äusseren Gehörgang vorwölbten.

Diese Untersuchungsergebnisse, welche mit den von Anderen bei ähnlichen Untersuchungen gewonnenen Resultaten im Grossen und Ganzen übereinstimmen, waren nicht so einheitlich, um sie zur sicheren Grundlage für weitere vergleichende Untersuchungen zu machen; denn wenn bei Leuten, die zur Zeit keineswegs abgemagert waren, vom äusseren Gehörgang aus ein deutliches Respirationsgeräusch wahrnehmbar war, so durfte man aus derselben Beobachtung, wenn man sie bei einem abgemagerten Kranken machte, nicht auf ein durch Schwund des Fettpolsters bedingtes pathologisches Offenstehen der Tuba schliessen; andererseits konnte bei einem solchen Kranken in der 'That die Tuba offen stehen, aber das Athmungsgeräusch war infolge verminderter Athmungsenergie vielleicht schwächer, als bei einem Gesunden, dessen Tuba normaler Weise so wenig fest geschlossen war, dass der Verschluss schon bei der Athmung gebrochen wurde. In gleicher Weise mussten diese Umstände auf das Ergebniss der Manometer- 
untersuchungen einwirken, ganz abgesehen davon, dass man schon aus̀ rein äusseren Grïnden bei hochgradig geschwächten Kranken, wenn man den Schwächezustand in gebuihrender Weise bericksichtigt, kaum ein ungetrïbtes Resultat erhält. Schliesslich mussten auch alle diejenigen Ursachen störend auf das Resultat der bei stark abgemagerten Personen angestellten Versuche einwirken, welche wir an früherer Stelle als Hindernisse für das Auftreten der Antophonie trotz theilweisen Schwundes des Fettpolsters aufführten. Nach mannigfachen Versuchen habe ich daher meine Bemühungen, nach dieser Richtung hin die Eröffnung der Tuba während starker Abmagerung nachzuweisen, als aussichtslos aufgegeben, zumal da zum Nachweis hierfür der beigebrachte pathologisch-anatomische Nachweis genïgen dürtte.

Es wäre nun sehr wünschenswerth gewesen, die vorstehenden Ausführungen durch Untersuchungen des Fettpolsters bei alten Leuten, bei denen physiologisch eine Fettritckbildung einzutreten pflegt, zu erweitern, um zu erfahren, ob das von $R$ ü $d$ in $g$ e $r^{1}$ ) und Wagenhäuser (l. c.) bei solchen beobachtete anomale Offenstehen der Tuba Eustachii auf physiologischem Schwund des Fettpolsters der lateralen Tubenwand zuruickzufuhren ist. Die Untersuchungen müssten naturgemäss an Tuben vorgenommen werden, welche hochbetagten Leuten entstammen, die infolge eines plötzlichen Todes eine dureh längere Krankheit bedingte Abmagerung nicht erfahren haben. Leider stand mir derartiges Material bisher nicht zur Verfügung and kann ich mich daher nur auf die Untersuchungen $R$ iudinger's (l. c.) stiitzen. Er fand bei 70-80jährigen Individuen beider Geschlechter häufig das Ostium tubae pharyngeum viel weiter, als bei Personen in mittleren Lebensjahren, und die Tuba in ihrer ganzen Ausdehnung weitklaffend. Der Musculus tensor veli palatini erschien sehr schwach, wenn nicht theilweise atrophirt, die Schleimhautfalten fehlten vollständig, der Knorpelhaken war etwas auswärts gerichtet, und an drei von ihm untersuchten Tuben fehite das an der lateralen Wand befindliche Fett fast vollständig. „Die Abweichung, welche das zeitweilige Offensein zur lästigen Empfindung macht, scheint $R$ ï inger in einem Klaffen, einer Erschlaffung, vielleicht in einer Atrophie der Tubenwände und Tubenmuskeln zu bestehen."

Bei alten Leuten kommt also in ganz ähnlicher Weise wie

1) Ueber anomales Offensein der Tuba Eustachii bei alten Leuten. Monatsschr. f. Ohrenheilk. Il. Jahrg. Nr. 9. 
während starker Abmagerung bei Personen in mittleren Lebensjahren ein fast vollständiger Sehwund des Fettpolsters an der lateralen Tubenwand vor, und ich stimme $R$ ü ding er vollkommen bei, soweit er die Eröffnung der Tuba bei alten Leuten hierauf, sowie auf die nachgewiesene Atrophie der Tubenschleimhant, möglicher Weise auch auf die Veränderung des Tubenkuorpels bezieht, kann mir jedoch nicht vorstellen, wie durch Atrophie der Tubenmuskeln, speciell des Dilatator tubae, die Tuba eröffnet werden soll. Wenn ein Muskel atrophirt, so verringert sich seine Kraft und vermindert sich, wenn wir von ganz besonderen Fällen absehen, sein Volumen. Wie soll nun der Dilatator tubae, der bei normalem Verhalten in der Ruhe die Tuba nicht eröffnet, dies fertig bringen, wenn er atrophirt? Sein auf den Tubenhaken bezw. die laterale Tubenwand direct ausgeubter Zug wird dann doch nur schwächer werden können, als friiher, und noch viel weniger eine dauernde Eröffnung der Tuba berbeiführen, wie bei normalem Verhalten. Indess sein Volumen verringert sich. Wenn man die herausgenommene Tuba von der lateralen Seite her betrachtet, so überdeckt der Dilatator tabae wie ein ganz diuner Vorhang die laterale Tubenwand und bietet ihr einen Stiutzpunkt und elastischen Widerhalt. Bei theilweiser Atrophie des Muskels wird sich dieser allerdings verringern können, doch dürfte dieser Umstand für die Eröffnung des Trabenlumens nur in Verbindung mit dem Schwund des Fettpolsters und der Schleimhautatrophie von unterstiitzender Bedeutung sein. Wenigstens kann man sich dies doch vorstellen.

Bei den ron mir untersuchten Tuben stark abgemagerter Personen habe ich weder Atrophie der Muskeln noch der Schleimhaut, deren Falten anf das Deutlichste ansgeprägt waren, gefunden, und ich bin der Ansicht, dass es beim Sehwund des Fettes im Allgemeinen um so leichter zu einem Offenstehen der Tuba kommen wird, je weniger die Kraft der Tubenerweiterer geschwächt ist.

Das Fettpolster der lateralen Tubenwand ist ein natürlicher Schutz für das Mittelohr insofern, als mit dureh dieses das Anliegen der lateralen an die mediale Tubenwand bedingt wird, und von seiner individuell wie zeitlich verschiedenen Entwicklung dürfte unter sonst vollkommen normalen Verhältnissen zum Theil der verschiedene Grad der Festigkeit des Verschlusses der Hülfsspalte abhängen. Der Schutz, den es gewährt, besteht nicht allein darin, dass das Mittelohr gegen das Eindringen der Schallwellen 
bei der Stimmgebung und gegen lästige Respirationsgeräusche gesichert ist, sondern auch das unmittelbare Hineingelangen infectiöser Stoffe vom Nasenrachenraum aus erschwert wird.

Die Wege, auf denen bei Infectionskrankheiten Mittelohrentziündungen entstehen können, sind mannigfaltig. Bald mag die Entzïndung vom Nasenrachenraum aus unmittelbar durch die Tuba fortgeleitet sein, bald mag sie als eine Theilerscheinung der Gesammtinfeetion des Organismus aufzufassen oder durch embolische Vorgänge zu erklären sein. Ein Bruchtheil dieser Mittelohrentzitndungen dürfte indess einem unmittelbaren Eindringen von Entzïndungserregern vom Nasenrachenratm aus seine Entstehung verdanken, und besonders mit Rücksicht auf dieses ätiologische Moment dürfte die Weite der Ohrtrompete keineswegs gleichgültig sein. Dass ein directes Hineinschleudern corpusculärer Elemente vom Cavum pharyngonasale aus in das Mittelohr möglich ist, lehren die Fälle, in denen bei unterdrücktem Niesen Schnupftabak hinein gerieth. Es ist immerhin beachtenswerth und spricht gewiss nicht gegen unsere Ansicht von der sehitzenden Wirkung des Fettpolsters, wenn wir aus den Zusammenstellungen Bezold's 1) über 1243 Typhuskranke erfahren, dass der Beginn der schweren eitrigen Mittelobrentzindungen beim Typhus nahezu 4 Proc. - durchschnittlich in die 4. und 5. Woche der Allgemeinerkrankung fällt, also in eine Zeit, wo der typhöse Process als solcher im Allgemeinen abgelaufen, die Abmagerung dagegen am stärksten zu sein pflegt, während die nervösen Affectionen des Gehörorgans in die Anfangs-, theilweise schon in die Prodromalperiode fallen, und wenn es sich zeigt, dass bei Tuberculose am häufigsten das $\mathrm{Ohr}$ erst in vorgeschritteneren Stadien der Allgemeinerkrankung in Mitleidenschaft gezogen wird. Ich selbst habe bei einem stark abgemagerten Phthisiker unter lebhafter Temperatursteigerung so plötzlich eine eitrige Mittelohrentzindung entstehen seben, dass ich mich nicht des Eindruckes erwehren konnte, es sei in diesem Falle bei unterdrückten Hustenstössen infectiöses Material vom Nasenrachenraum ans direct in das Ohr hineingeworfen worden.

Das Ergebmiss der Untersuchung lässt sich dahin zusammenfassen:

1. Das Fettpolster der lateralen Tubenwand bildet eine Sehutzvorrichtung für das Mittelohr.

1) Ueber die Erkrankungen des Gehörorgans bei lleotyphus. Archiv f: Ohrenheilk. Bd. XXI. S. 8. 
2. Bei Abmagerung verschwindet dasselbe in mehr oder weniger ausgedehntem Maasse.

3. Durch diesen Fettschwund wird die zuweilen beobachtete zeitweilige Eröffnung der Tuba bei stark abgemagerten Personen erklärt.

4. Eine kräftige Wirkung der Tubenmuskeln, insbesondere des Musculus dilatator tubae, sowie eine directe Verbindung desselben mit der lateralen, häutigen Wand kann die zeitweilige Eröffnung der Tuba nur fördern. 